\title{
UOCS. IV. Discovery of diverse hot companions to blue stragglers in the old open cluster King 2
}

\author{
VIKRANT V. JADHAV ${ }^{1,2, *\left(\mathbb{D}, \text { SINDHU PANDEY }^{3} \text { (D), ANNAPURNI SUBRAMANIAM }\right.}{ }^{1}$ \\ and RAM SAGAR ${ }^{1,3}$ \\ ${ }^{1}$ Indian Institute of Astrophysics, Sarjapur Road, Koramangala, Bangalore 560 034, India. \\ ${ }^{2}$ Joint Astronomy Programme and Department of Physics, Indian Institute of Science, Bangalore 560012 , \\ India. \\ ${ }^{3}$ Aryabhatta Research Institute of Observational Sciences, Manora Peak, Nainital 263 002, India. \\ *Correspondence author. E-mail: vikrant.jadhav@iiap.res.in
}

MS received 7 November 2020; accepted 15 February 2021

\begin{abstract}
King 2, one of the oldest clusters in the Milky Way, with an age of $\sim 6$ Gyr and distance of $\sim 5700 \mathrm{pc}$, has been observed with UVIT payload on the AstroSat. With membership information derived from Gaia EDR3, the cluster is found to have 39 blue straggler stars (BSSs). We created multi-wavelength spectra-energy distributions (SED) of all the BSSs. Out of $10 \mathrm{UV}$ detected BSSs, 6 bright ones were fitted with double component SEDs and were found to have hotter companions with properties similar to extreme horizontal branch $(\mathrm{EHB}) /$ subdwarf $\mathrm{B}(\mathrm{sdB})$ stars, with a range in luminosity and temperature, suggesting a diversity among the hot companions. We suggest that at least $15 \%$ of BSSs in this cluster are formed via mass-transfer pathway. When we compared their properties to EHBs and hotter companions to BSS in open and globular clusters, we suggest that $\mathrm{EHB} / \mathrm{sdBs}$ like companions can form in binaries of open clusters as young as 6 Gyr.
\end{abstract}

Keywords. Open star clusters (1160)—blue straggler stars (168)—extreme horizontal branch stars (513) — B subdwarf stars (129)—ultraviolet astronomy (1736)—spectral energy distribution (2129)—binary stars (154).

\section{Introduction}

The evolution of binary systems strongly depends on the initial orbital parameters and its further evolution, where any change in their orbits can lead to a widely different evolution. If one of the stars evolves and fills its Roche lobe, the system will undergo mass transfer. Details such as duration and rate of mass transfer will depend on the orbits and masses of the binary stars. If such a binary is present in a star cluster, and the secondary of the binary has mass similar to the main sequence turnoff (MSTO) mass, then the secondary will become brighter than the MSTO and appear as a

This article is part of the Special Issue on "AstroSat: Five Years in Orbit". blue straggler star (BSS). Otherwise, the secondary will be fainter than the MSTO and can be classified as a blue lurker. The blue lurkers are identified by their high stellar rotation (Leiner et al. 2019) or evidence of extremely low-mass (ELM) white dwarf (WD) companion (Jadhav et al. 2019). Depending on the evolutionary status of binary components, the binary system can be observed as main sequence (MS)+MS, contact binaries (Rucinski 1998), common envelop, MS+horizontal branch (HB; Subramaniam et al. 2016), MS+extreme HB (EHB; Singh et al. 2020), MS+ subdwarf-B (sdB; Han et al. 2002), MS+WD (Jadhav et al. 2019), WD+WD (Marsh et al. 1995) and many more combinations. The binary evolution also depends on external factors such as collisions in a high-density environment which can decouple the binary (Heggie 1975) and 
tertiary star which can expedite the mass transfer/ merger by reducing the orbital separation (Kozai 1962).

We are carrying out a long term project ${ }^{1}$ of characterising products of binary stars such as BSSs in open clusters (OCs). Ultraviolet imaging of binary systems reveals the presence of hotter companions in the binary system, given that the hotter companion is luminous in UV. Old OCs such as NGC 188 and NGC 2682 are rich with BSSs, binary stars and contain many such optically sub-luminous UV-bright companions (Subramaniam et al. 2016; Sindhu et al. 2019; Jadhav et al. 2019). Similar companions have been identified to BSSs in the outskirts of GCs (Sahu et al. 2019; Singh et al. 2020). Subramaniam et al. (2020) provides a summary of the BSSs and post mass transfer systems in star clusters.

King 2 is one of the oldest clusters in the Milky Way, with an age of $\sim 6$ Gyr and distance of $\sim 5700$ pc (Table 1). However, it has been poorly studied due to its considerable distance and unknown membership information. For identifying and characterising hot BSSs and their possible companions, we obtained Ultra Violet Imaging Telescope (UVIT)/AstroSat observations of rich OC King $2\left(\alpha_{2000}=12.75 ; \delta_{2000}\right.$ $=+58.183 ; l=122.9$ and $b=-4.7)$ under AstroSat proposal A02_170. Kaluzny (1989) presented the first optical colour-magnitude diagram (CMD) study of this distant cluster using $B V$ CCD photometric data. This yielded a range of plausible ages and distances for different assumed reddenings and metallicities. The galactocentric distance of the cluster was estimated to be $\sim 14 \mathrm{kpc}$. Aparicio et al. (1990) (A90 hereafter) did a comprehensive study on the cluster using $U B V R$ photometry and derived an age of $6 \mathrm{Gyr}$ and a distance of $5.7 \mathrm{kpc}$ for solar metallicity. They also indicated the presence of a good fraction of binaries in the MS. Tadross (2001) estimated a value of $[\mathrm{Fe} / \mathrm{H}]=-0.32$ using the $(U-B)$ colour excess from the literature data, while Warren and Cole (2009; WC09 hereafter) derived a value of $[\mathrm{Fe} / \mathrm{H}]=-0.42 \pm$ 0.09 using spectroscopic data. These metallicity estimates are significantly sub-solar and inconsistent with the finding of A90. WC09 found a distance of $6.5 \mathrm{kpc}$ and a slightly younger age, $\sim 4 \mathrm{Gyr}$, better fitted the optical CMD and 2MASS Ks, red clump if the reddening is adopted as $E(B-V)=0.31$ mag. This distance puts King 2 at $\mathrm{RGC}=13 \mathrm{kpc}$, where its metallicity falls close to the trend of the galactic abundance gradients derived in Friel et al. (2002). There has been no proper motion study available for this cluster till Gaia DR2 (Gaia Collaboration et al. 2018). Cantat-Gaudin et al. (2018) provided a membership catalogue of King 2 with 128 members with Gaia DR2, and Jadhav et al. (2021) provided kinematic membership of 1072 stars (and 340 probable members) using kinematic data taken from Gaia EDR3.

Above discussed optical photometric studies indicate a good number of post-MS hot stars in King 2. In fact, Ahumada and Lapasset (2007) have identified 30 BSS candidates based on the location of these stars in the cluster. We present the UVIT and the archival data used in this study in the next section, followed by analyses, results and discussion.

\section{UVIT and archival data}

We observed King 2 with UVIT, which is one of the payloads on the first Indian multiwavelength space observatory AstroSat, launched on 28 September 2015. The observation was carried out by UVIT on 17 December 2016, simultaneously in two filters. The telescope has three channels with a set of filters in them: Far-UV (FUV; 130-180 nm), near-UV (NUV; 200-300 nm) and visible (VIS; $350-550 \mathrm{~nm}$ ), where the VIS channel is intended to correct the drift of the

Table 1. Age, distance, reddening $(E(B-V))$ and metallicity of King 2 estimated by various investigators are listed.

\begin{tabular}{lllll}
\hline $\begin{array}{l}\text { Age } \\
(\mathrm{Gyr})\end{array}$ & $\begin{array}{c}\text { Distance } \\
(\mathrm{pc})\end{array}$ & $\begin{array}{c}E(B-V) \\
(\mathrm{mag})\end{array}$ & Metallicity & \multicolumn{1}{c}{ References } \\
\hline 6.02 & 5750 & 0.31 & -0.42 & $\begin{array}{l}\text { Dias } \text { et al. } \text { (2002) } \\
\text { Aparicio } \text { et al. (1990) } \\
6\end{array}$ \\
$5690 \pm 65$ & $0.31 \pm 0.02$ & $\begin{array}{l}-0.5 \text { to }-2.2 \\
\text { Tadross (2001) }\end{array}$ \\
4 to 6 & $\sim 7000$ & 0.23 to 0.5 & -0.32 & Kaluzny (1989) \\
\hline
\end{tabular}

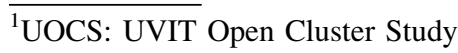



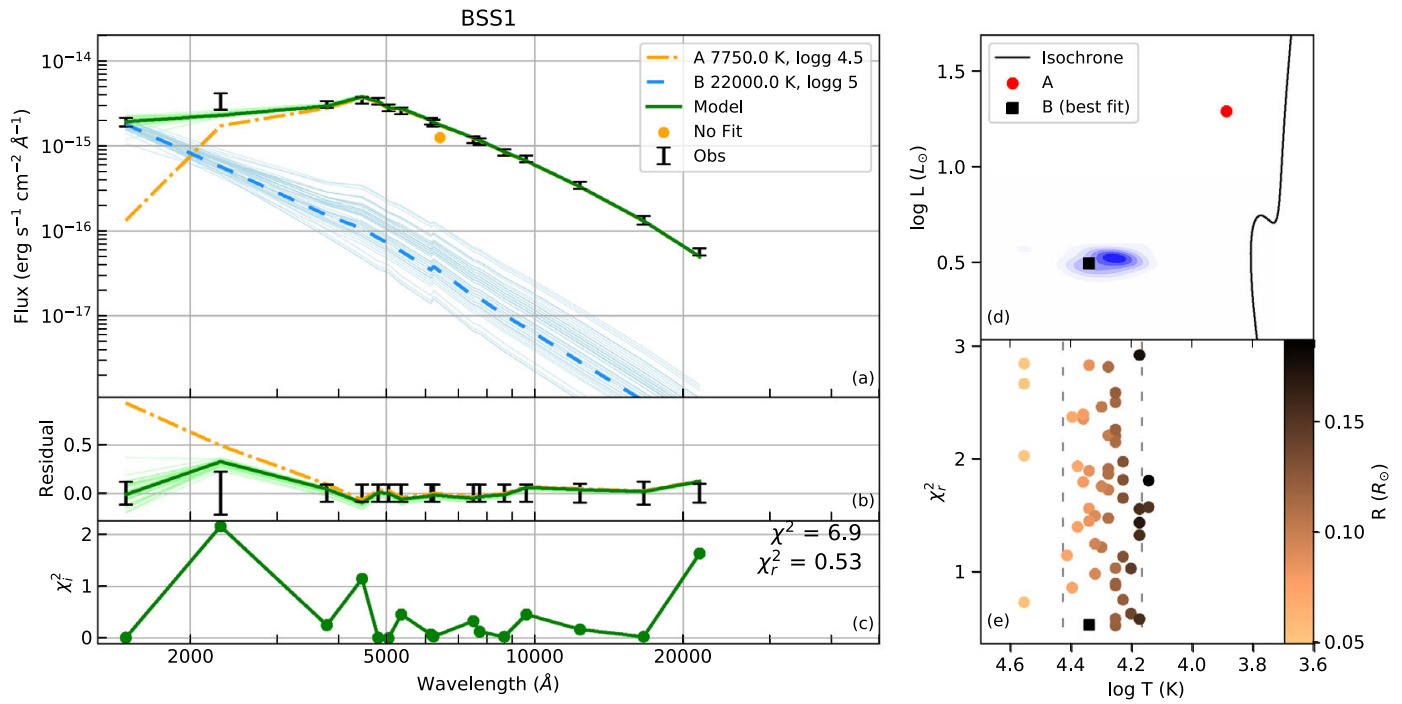

Figure 1. Two-component SED of BSS1. (a) Composite SED (green curve) is shown along with the observed flux (as black error-bars). The unfitted point (in this case: CAHA.R) is shown as orange dot. The cooler (BSS, orange dot-dashed curve) and hotter (blue dashed curve) component are also shown with their $T_{\text {eff }}$ and $\log g$. The model, B component and residuals of noisy iterations are also shown as light coloured lines. (b) The fractional residual is shown for single component fit (orange dot-dashed curve) and composite fit (green solid curve). The fractional observational errors are also indicated on X-axis. (c) The $\chi_{i}^{2}$ of each data point. (d) $\mathrm{H}-\mathrm{R}$ diagram of the two components along with the isochrone for reference. The density distribution of the noisy B component fits is plotted in blue. (e) $T_{\text {eff }}-\chi^{2}$ distribution for the noisy B component fits coloured according to their radii. The dashed lines are the quoted limits of the temperature.

spacecraft (see Kumar et al. 2012; Tandon et al. 2017 for more details). The cluster was observed in one FUV (F148W, limiting magnitude $\approx 23 \mathrm{mag}$ ) and one NUV (N219M, limiting magnitude $\approx 22 \mathrm{mag}$ ) filter for exposure time of $\sim 2.7 \mathrm{ks}$. The FWHM of PSF in F148W and F219N images is $1^{\prime \prime} .33$ and $1^{\prime \prime} .35$ respectively. The data reduction was done using CCDLAB (Postma \& Leahy 2017) and PSF photometry was performed using DAOPHOT package of IRAF (Tody 1993). More details of the reduction process are presented in Jadhav et al. (2021). We have detected ten member stars in either F148W and/or N219M filter.

We obtained archival optical $(U B V R)$ photometry data from A90 catalogue (Calar Alto Observatory; CAHA) and cross-matched with UVIT data using TOPCAT (Taylor 2005). The cluster was observed with GALEX under All-sky Imaging Survey (AIS) in NUV filter (exp. time $\sim 100 \mathrm{~s}$ ). All the detected member stars were further cross-matched with photometric data from UV to IR wavelength bands obtained from GALEX (Bianchi et al. 2000), PANSTARRS PS1 (Chambers et al. 2016), Gaia EDR3 (Gaia Collaboration et al. 2020), 2MASS (Skrutskie et al. 2006), WISE (Wright et al. 2010) using virtual observatory tools in VOSA (Bayo et al. 2008).

\section{SED fitting and colour magnitude diagrams}

The data were corrected for reddening $(E(B-V)=$ $0.31 \pm 0.02)$ using Fitzpatrick (1999) and Indebetouw et al. (2005) and calibrated with the cluster distance of $5750 \pm 100 \mathrm{pc}$ (we have overestimated the error to cover distance estimates from Dias et al. (2002) and A90). We have adopted the metallicity of $[\mathrm{Fe} / \mathrm{H}]=$ -0.5 for all the stars and used Kurucz model spectrum (Castelli et al. 1997) for comparison. The SED fitting was done as follows:

(1) We constructed the observed spectral energy distribution (SED) for all stars using the data from UV to IR wavelength, as mentioned above. The fluxes of all stars are given in Table 3.

(2) Kurucz models (Castelli et al. 1997) of $\log g \in$ $(3.0,5.0)$ were fitted to optical and IR points (above $3000 \AA$ ) using $\operatorname{VOSA}^{2}$ (Bayo et al. 2008). There were some sources which showed UV excess in multiple UV points compared to the model fit. We selected such stars to be fitted with a two component SED. Otherwise, the single component fits are deemed satisfactory and are stated in the lower part of Table 2.

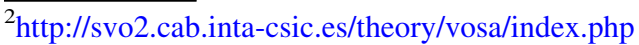


(3) We used the cooler component parameters from above fits and then fitted a hotter component to the residual using Binary_SED_Fitting ${ }^{3}$. In preliminary double component fits, the hotter components were found to be compact objects, hence they were fitted with $\log g=5$ Kurucz models.

(4) Very small errors in PAN-STARRS PS1, Gaia EDR3 and A90 photometry led to ignoring relatively high error UV data-points, hence they were replaced with mean errors for better residual across all wavelengths. A few data points were removed to achieve better fits and lower $\chi^{2}$ (see Fig. 1(a)). The error values given in Table 3 are original unmodified errors. The detailed SED fitting method is explained in Section 3.3 of Jadhav et al. (2019).

(5) The best-fit parameters for single stars or cooler components are taken from the VOSA fits. The hotter component parameters are taken from the least $\chi^{2}$ model in the two component fitting.

(6) The errors in cooler component parameters are fairly low and are taken as the grid values. To derive errors in the hotter component parameters, we used a statistical approach. We first generated 100 iterations of observed SEDs with added Gaussian noise in each data point. These 100 SEDs were then fitted with double components. However, not all the double fits converged, hence we only kept hotter components with $6000 \mathrm{~K}<T_{\text {eff }}<37000 \mathrm{~K}$. Logarithmic distributions of the parameters from the noisy and converging iterations were then fitted with Gaussian distributions. FWHM of these Gaussian distributions are defined as the upper and lower limits of the fitting parameters (temperature, radius and luminosity).

Figure 2 shows the CMD of 1412 cluster candidates identified from Gaia EDR3 with probability of over 50\% (Jadhav et al. 2021) and are marked as grey points. Among these stars, we have selected 39 member stars brighter $(G<17.5 \mathrm{mag})$ and bluer $\left(G_{\mathrm{BP}}-G_{\mathrm{RP}}<1.1 \mathrm{mag}\right)$ than the MSTO as BSSs. Seven of them were detected in F148W, and seven were detected in $\mathrm{N} 219 \mathrm{M}$ (four in both filters). An isochrone of $\log$ age $=9.7$ is over-plotted on the CMD. 20 of the BSSs are detected in the NUV filter of GALEX. We fitted Kurucz model SEDs to all BSSs and found excess UV flux in 15 BSS (BSS 1, 2, 3, 4, 5, $7,8,9,10,19,26,28,29,33$ and 36). Among these,

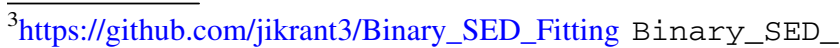
Fitting is a python code which uses $\chi^{2}$ minimisation technique to fit two component SEDs.

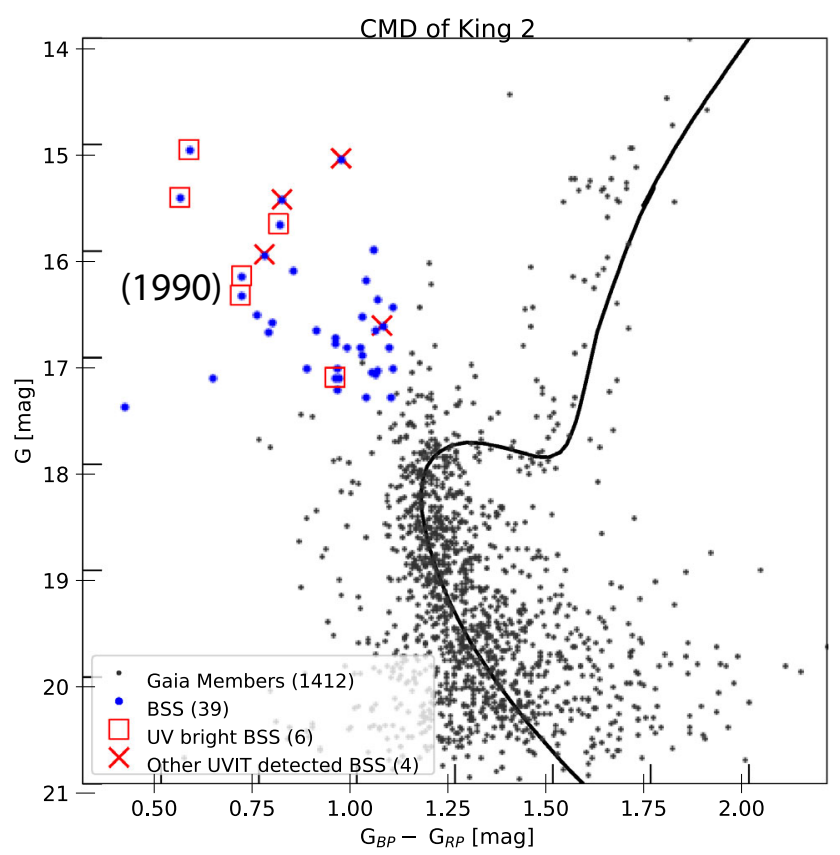

Figure 2. CMD of King 2 cluster candidates using Gaia EDR3 data. All BSS members are shown as blue circles. The UV bright BSS (see Section 3) are shown as red squares, and other UVIT detected BSS are shown as red X's. The Gaia members are shown as black dots along with the PARSEC isochrone of log age $=9.7,[\mathrm{M} / \mathrm{H}]=-0.4, \mathrm{DM}$ $=13.8$ and $E(B-V)=0.45$.

BSS1, 2, 3, 4, 5 and 7 have multiple UV data points from UVIT or GALEX or both. Only these six were fitted with double component SEDs, because a hot component fit can be reliable if the number of UV data points is more than one. Hereafter, these six BSS will be referred to as 'UV bright BSSs' and others will be referred to as 'UV faint BSSs'. The four BSS detected in UVIT but not fitted with hotter component are shown as red X's in the CMD.

We have shown an example of a double component SED fit of BSS1 in Fig. 1(a). The BSS1-A component is a BSS with $7750 \mathrm{~K}$, while the BSS1-B component has $T_{\text {eff }}$ of $22000 \mathrm{~K}$. The reduction in residual after including the hotter component is visible in Fig. 1(b). The $\chi_{i}^{2}$ for individual points is shown in Fig. 1(c) with $\chi_{r}^{2}$ of 0.53 . Although, we note that the $\chi_{r}^{2}$ need not be $\sim 1$, for a non-linear model fitting (Andrae et al. 2010). One has to look at residuals and $\chi^{2}$ both to determine the goodness of fit. Figure 1(d) shows the Hertzsprung-Russell (H-R) diagram of A and B components. The density distribution of noisy and converging iterations is also shown to get an idea of degeneracy in temperature and luminosity. Figure 1(e) panel shows the best fit and the noisy and converging 
Table 2. Fitting parameters of the best fit of the double and single component fits of BSSs with the hotter component. Scaling factor is the value by which the model has to be multiplied to fit the data, $N_{\text {fit }}$ is the number of data points fitted and $\chi_{r}^{2}$ is the reduced $\chi^{2}$ for the composite fit. The $\chi_{r}^{2}$ values of single fits of the cooler components are given in brackets. Note: the $\log g$ values are imprecise due to the insensitivity of the SED to $\log g$.

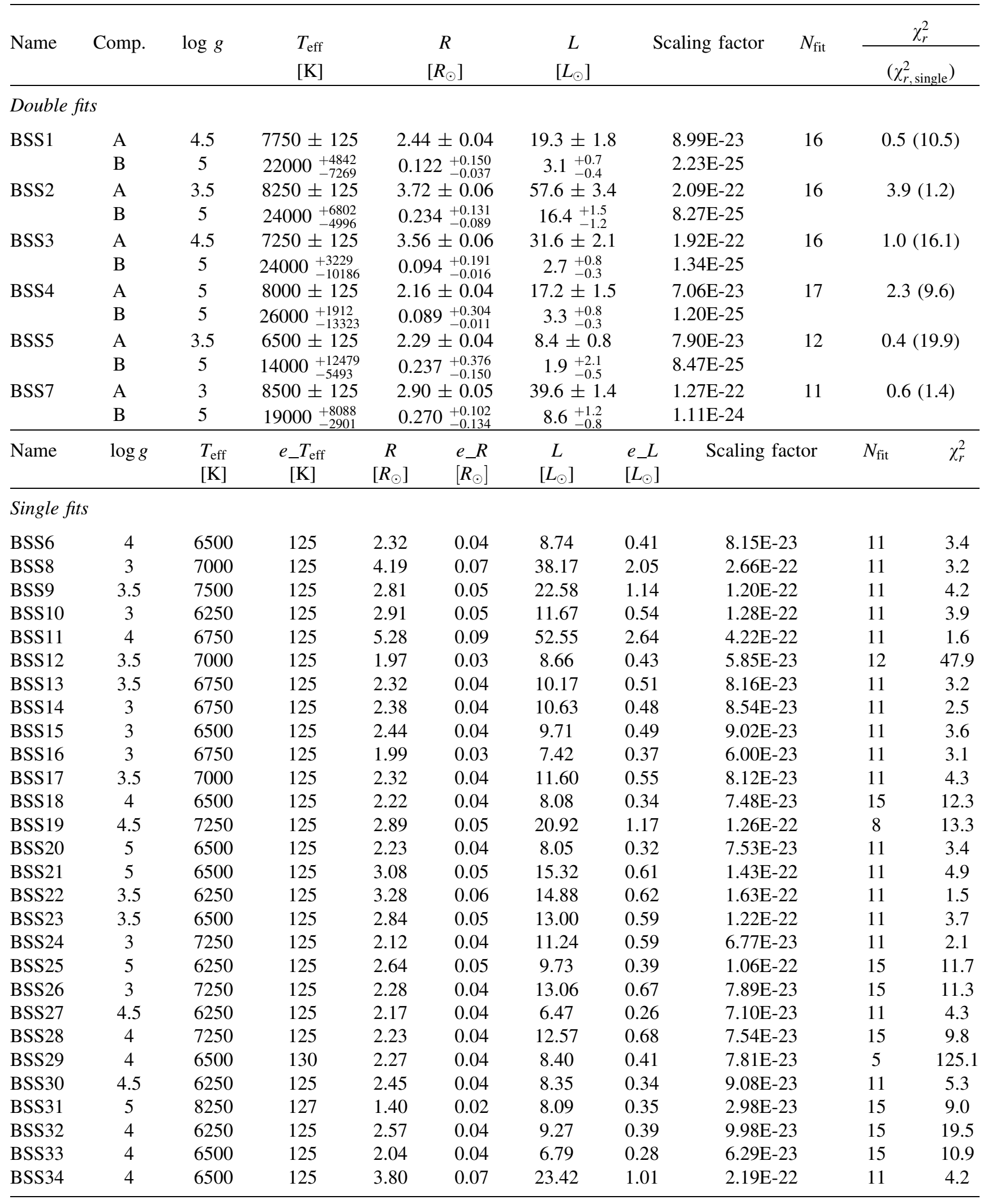


Table 2. Continued.

\begin{tabular}{|c|c|c|c|c|c|c|c|c|c|c|}
\hline Name & $\log g$ & $\begin{array}{l}T_{\text {eff }} \\
{[\mathrm{K}]}\end{array}$ & $\begin{array}{c}e_{-} T_{\text {eff }} \\
{[\mathrm{K}]}\end{array}$ & $\begin{array}{c}R \\
{\left[R_{\odot}\right]}\end{array}$ & $\begin{array}{l}e_{-} R \\
{\left[R_{\odot}\right]} \\
\end{array}$ & $\begin{array}{c}L \\
{\left[L_{\odot}\right]}\end{array}$ & $\begin{array}{c}e_{-} L \\
{\left[L_{\odot}\right]}\end{array}$ & Scaling factor & $N_{\text {fit }}$ & $\chi_{r}^{2}$ \\
\hline BSS35 & 3 & 6250 & 125 & 2.41 & 0.04 & 8.07 & 0.38 & $8.75 \mathrm{E}-23$ & 11 & 4.4 \\
\hline BSS36 & 3.5 & 6500 & 125 & 2.37 & 0.04 & 9.13 & 0.44 & $8.51 \mathrm{E}-23$ & 11 & 3.3 \\
\hline BSS37 & 5 & 6500 & 125 & 2.25 & 0.04 & 8.23 & 0.34 & 7.65E-23 & 11 & 3.6 \\
\hline BSS38 & 5 & 6500 & 125 & 2.68 & 0.05 & 11.70 & 0.48 & $1.09 \mathrm{E}-22$ & 11 & 3.4 \\
\hline BSS39 & 3.5 & 6500 & 125 & 3.31 & 0.06 & 17.67 & 0.80 & $1.65 \mathrm{E}-22$ & 11 & 3.9 \\
\hline
\end{tabular}

iterations in $T_{\text {eff }}-\chi^{2}$ phase-plane. The double component fits of BSS2, 3, 4, 5 and 7, and single-component fits of BSS10 and BSS15 are shown in Fig. 3. The fitting parameters are mentioned in Table 2.

The H-R diagram of the BSSs detected in King 2 is shown in Fig. 4. We have shown the UV faint BSSs as blue dots, UV bright BSSs are represented as blue diamonds, and the hotter components of UV bright BSSs as filler circles. We have taken the parameters of the hotter companions detected along with the BSSs in NGC 188 from Subramaniam et al. 2016 and NGC 2682 from Sindhu et al. (2019), Jadhav et al. (2019) and Sindhu et al. (in prep.). They are plotted in the figure as orange cross and triangles respectively. The parameters of the EHB stars in NGC 1851 are taken from Singh et al. (2020) and are shown as orange stars. The PARSEC ${ }^{4}$ isochrone of log age $=9.7$ is over-plotted and shown in black (Bressan et al. 2012), along with the WD cooling curves $^{5}$ (thick grey curves, Tremblay \& Bergeron 2009) and $\mathrm{BaSTI}^{6}$ zero age HB (ZAHB; dashed black curve; Hidalgo et al. 2018).

\section{Results and discussion}

\section{BSS and their companions in literature}

The BSSs have $T_{\text {eff }}$ range of 5750 to $8500 \mathrm{~K}$ and radii of 1.4 to $5.21 R_{\odot}$. By comparison to isochrones, they have mass in the range of 1.2 to $1.9 M_{\odot}$, the brightest BSS being 3 mag brighter than the MSTO. Majority of BSSs in King 2 have $T_{\text {eff }}$ similar to the older NGC 188 (6100-6800 K; Gosnell et al. 2015), but are cooler than NGC 2682 (6250-9000 K; Sindhu et al. in prep.), which is expected due to its slightly younger age. The BSSs in NGC 188 (Geller \& Mathieu 2011; Gosnell et al. 2014; Subramaniam et al. 2016) and NGC 2682

\footnotetext{
$\overline{{ }^{4} \text { http://stev.oapd.inaf.it/cgi-bin/cmd }}$

5 www.astro.umontreal.ca/ bergeron/CoolingModels/

${ }^{6}$ http://basti-iac.oa-abruzzo.inaf.it/isocs.html
}

(Sindhu et al. 2019) are known to have evolved companions. The companions were classified as WD, ELM WDs, post-AGB/HB according to their luminosity and temperature.

BSS2-A, BSS3-A and BSS7-A lie above/on the ZAHB in Fig. 3. There is a degeneracy in this region of the $\mathrm{H}-\mathrm{R}$ diagram where one could find both massive BSS as well as ZAHB stars. Stars in these two evolutionary phases will have different masses (HB mass < MSTO; BSS mass > MSTO), that could be used to lift the degeneracy. Bond and Perry (1971) measured the masses of stars in this region of the NGC 2682 CMD and determined that they are indeed high mass BSSs. One star is found in this region of the NGC 188 CMD and it is classified as a BHB (Rani et al. 2021), this star is significantly brighter than the rest of the BSSs. In the case of King 2, the BSSs show a continuous distribution up to the brightest BSS, hence BSS2-A/BSS3-A are most likely normal BSSs. However, their mass estimations (via $\log g$ measurements or asteroseismology) are required before confirming their evolutionary status.

\section{What are the hotter companions?}

The hotter companions in UV bright BSSs have $T_{\text {eff }}$ of 14000 to $26000 \mathrm{~K}$ (spectral type B) and radii of 0.09 to $0.27 R_{\odot}$. Figure 3 shows the density distributions of the best 100 fits for the hotter companions. Figure 3 also shows the location of various companions to BSS in NGC 188 and NGC 2682, and EHB stars in NGC 1851, one of which has a BSS as its companion (Singh et al. 2020).

The hotter companion to BSSs in NGC 2682 are all fainter and near the WD cooling curves. While those for NGC 1851 and NGC 188 lie closer to the ZAHB region. In King 2, the limiting magnitude of UVIT observations is 23 and 22 mag in F148W and N219M, respectively. According to WD cooling models (corrected for distance and extinction; Tremblay \& Bergeron 2009), only WDs younger than 0.7 and 16 


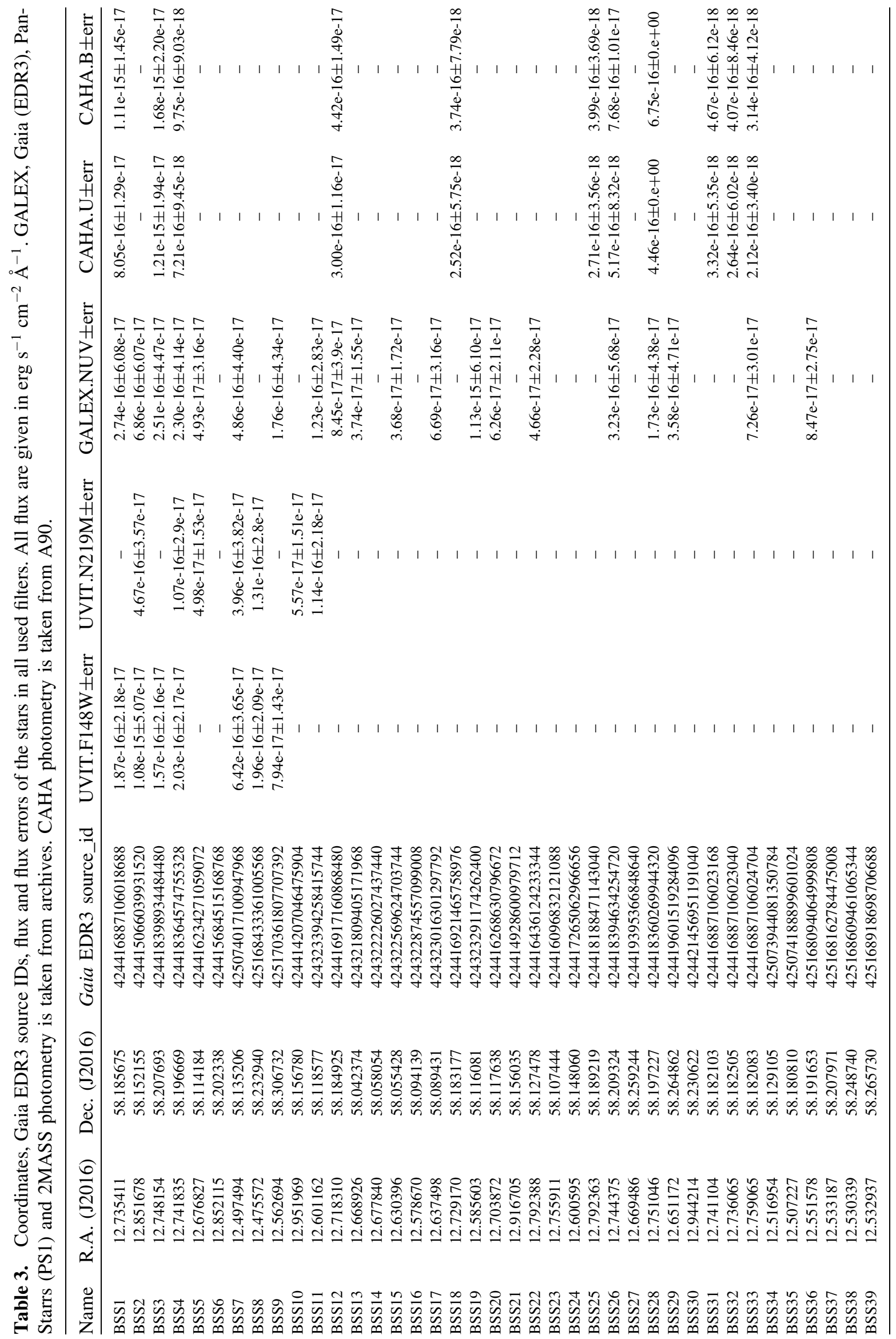




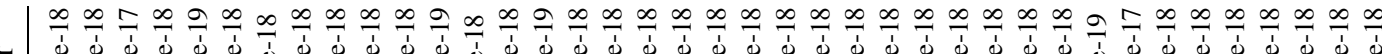

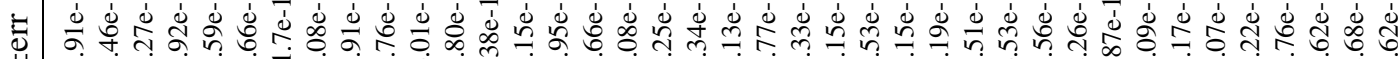

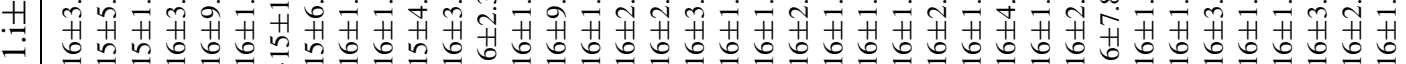

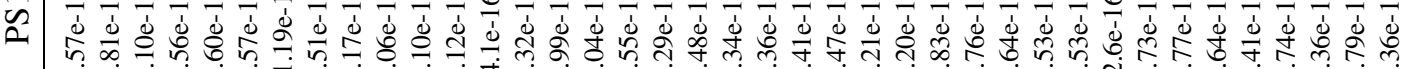

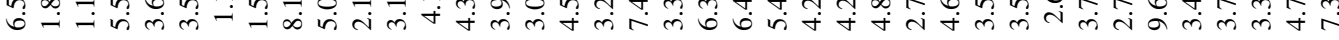

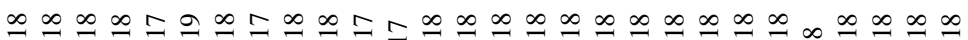

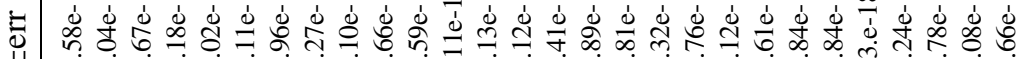

$\stackrel{\infty}{\rightarrow} \stackrel{\infty}{\sim} \cong \infty \infty ⿻$

Ho

$\overline{\check{\Omega}}$ ป

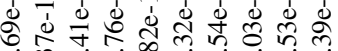

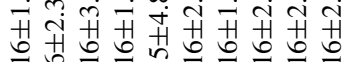
-

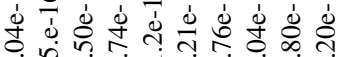

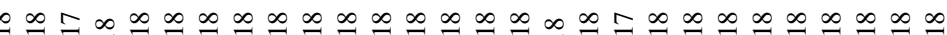

可

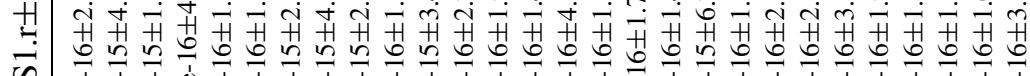

$\bar{n}$ ळ

$\infty \infty \infty \infty \infty \infty \infty \infty$

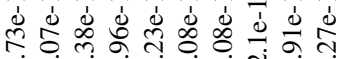

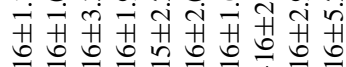
क त-

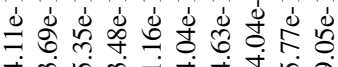

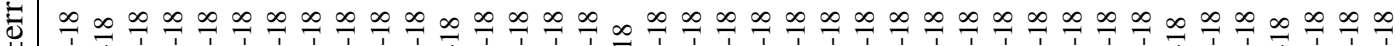
H

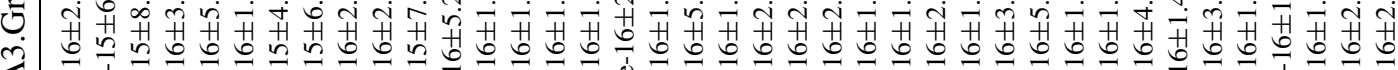

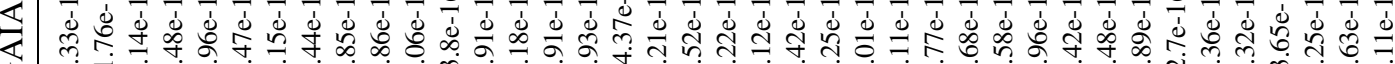
- - in

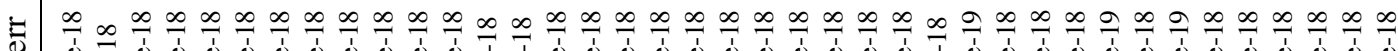

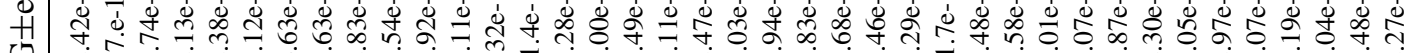

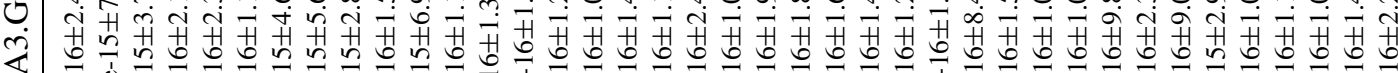

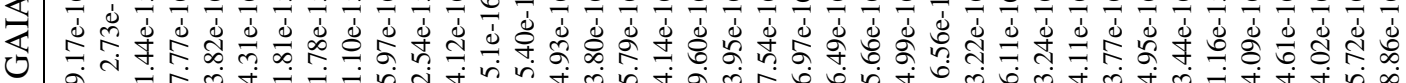

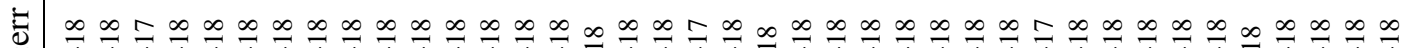

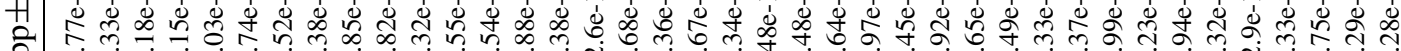

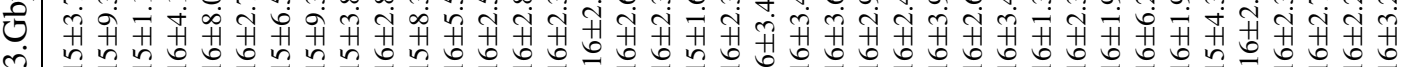

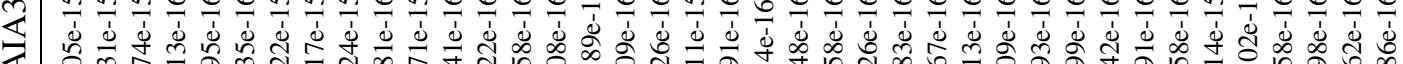

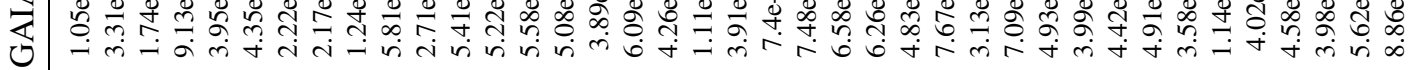

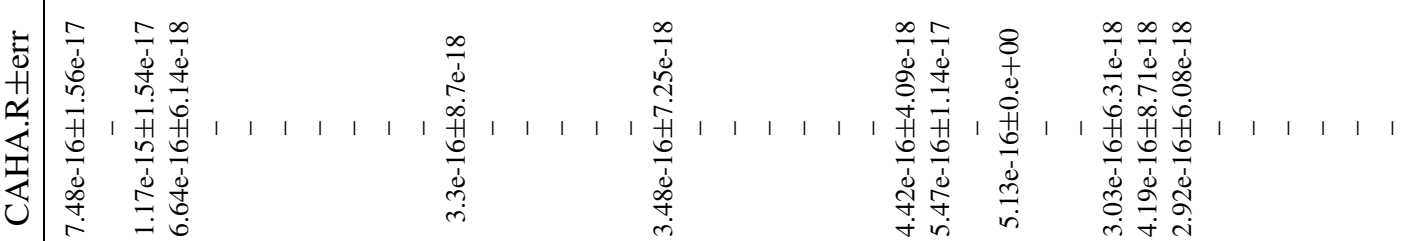

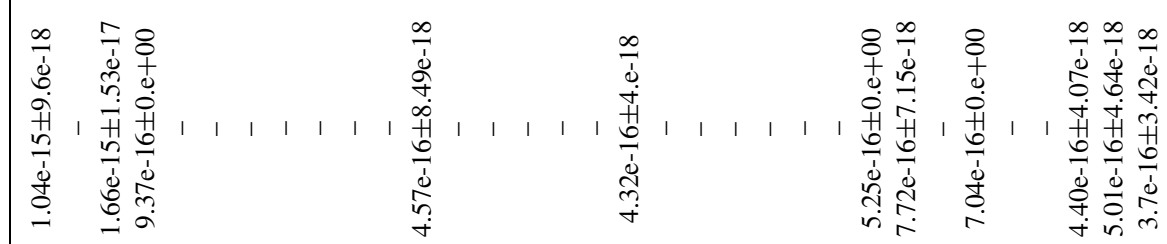




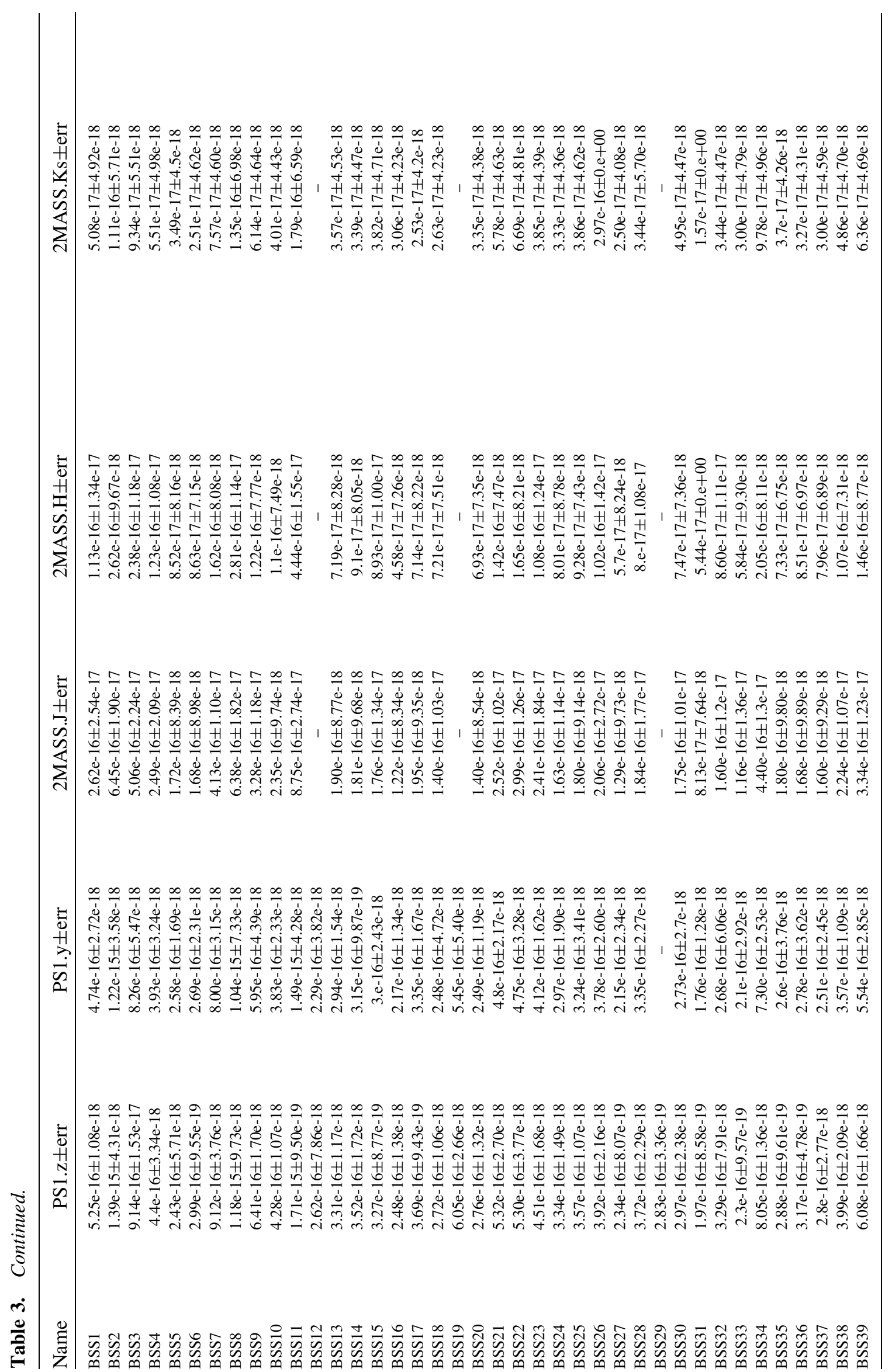



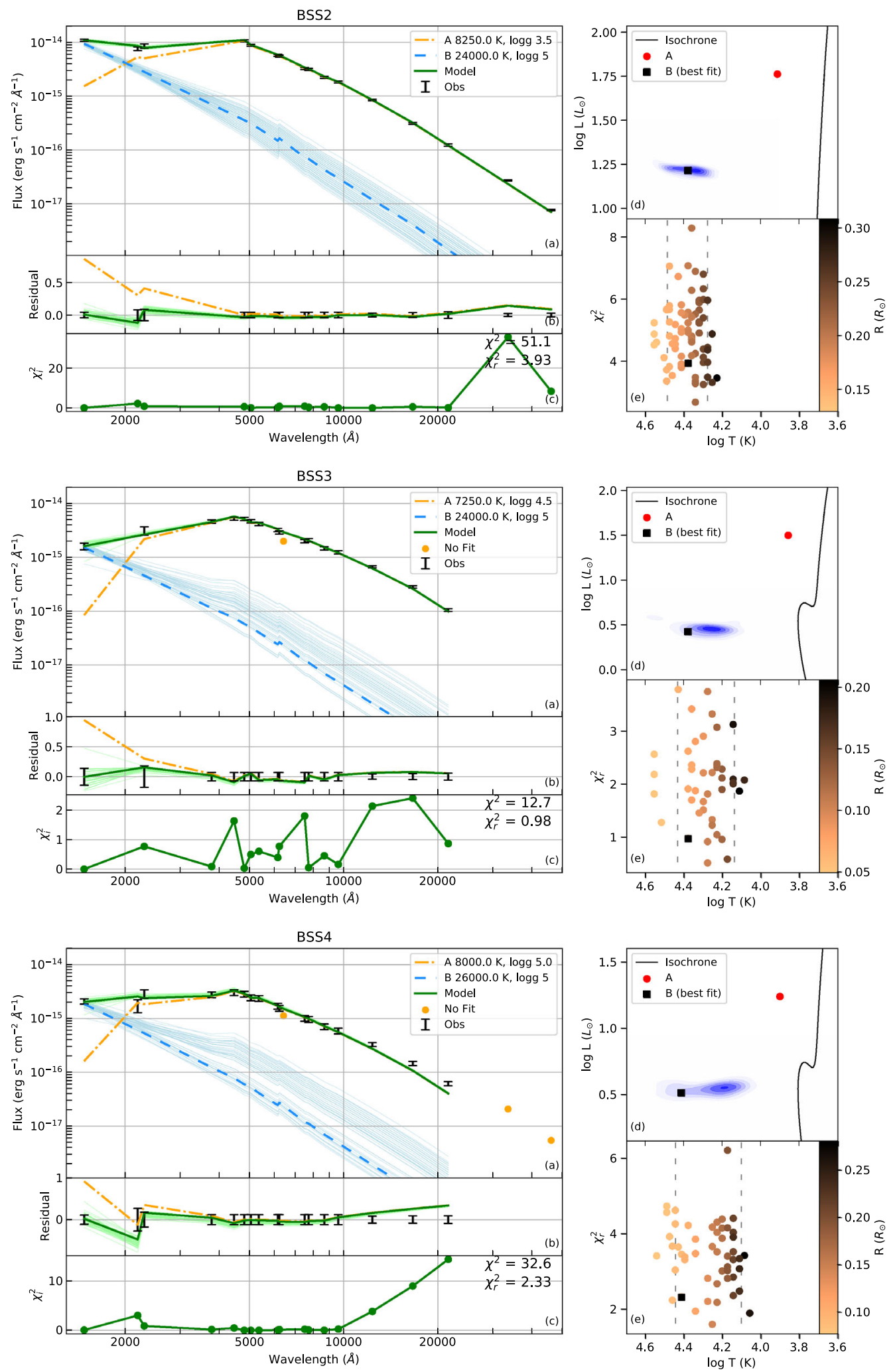

Figure 3. The descriptions of double component fits are same as Fig. 2. The single component fits of BSS10 and BSS15 are shown as an example with model fit (blue curve), fitted data points (red points) with $1 \sigma$ and $3 \sigma$ errors as solid and dashed lines. The theoretical spectra (in grey) is added for reference. The observed (reddening affected) SED is shown in grey below the corrected data-points. The title mentions the $T_{\text {eff }}, \log g$, metallicity and $A_{V}$ of the model fit. 

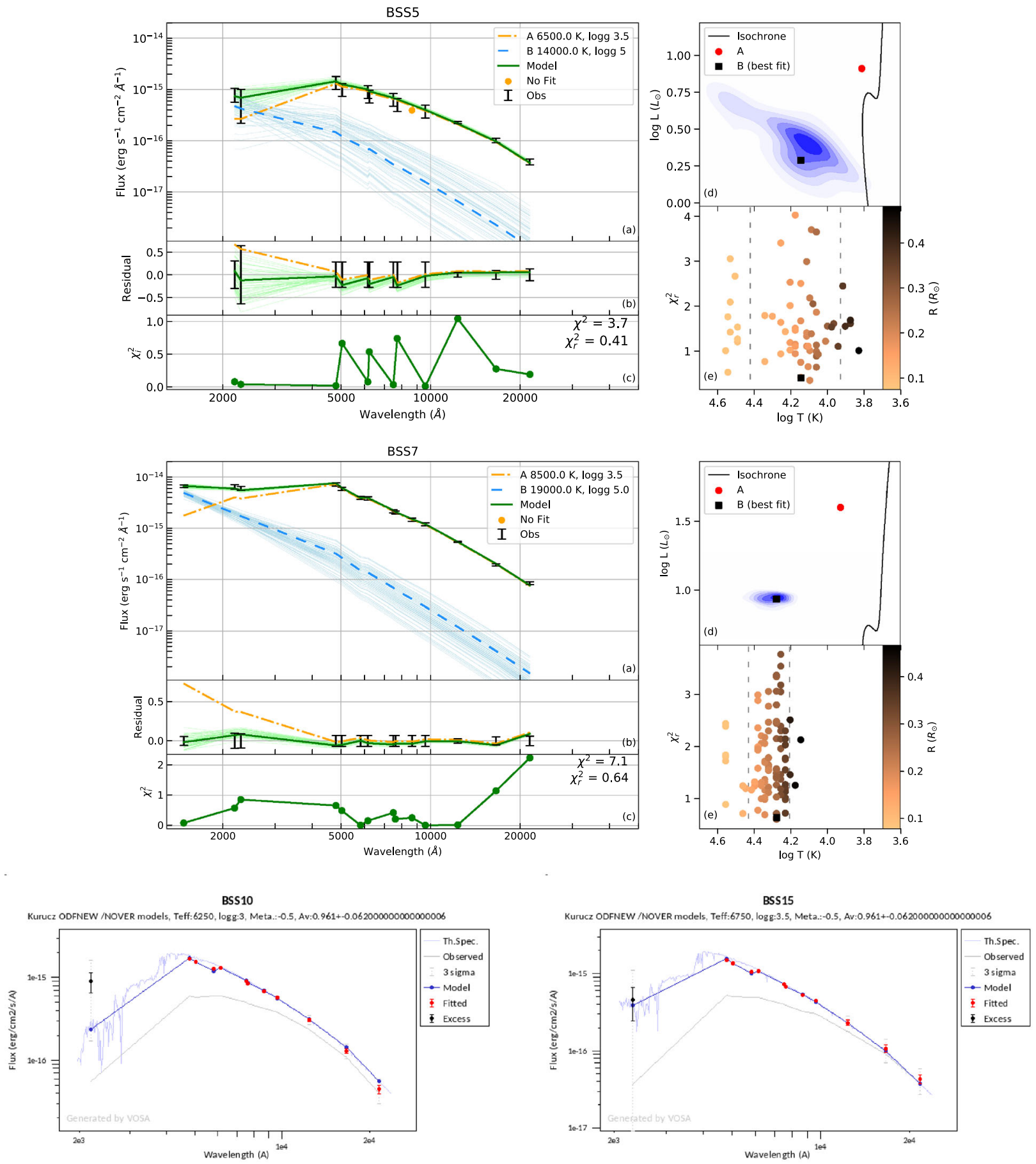

Figure 3. Continued.

Myr old would be detectable. As seen in Fig. 4, the hotter companions are well above the WD cooling curves, and these are not WDs.

The hotter companions are likely to be hot HB stars which are also known as EHB stars or sdB stars, as inferred from their $T_{\text {eff }}$, radii and luminosity. These are core-helium burning stars with $T_{\text {eff }}$ in the range of 20,000-40,000 $\mathrm{K}$ and are compact (0.15-0.35 $R_{\odot}$; Heber 2016; Sahoo et al. 2020). As these stars are hot and not as small as WDs, they appear bright in the UV. These stars are thought to contribute to the UV upturn seen in elliptical or in early-type galaxies (Brown et al. 1997). The sdB stars have very thin hydrogen envelope and are 


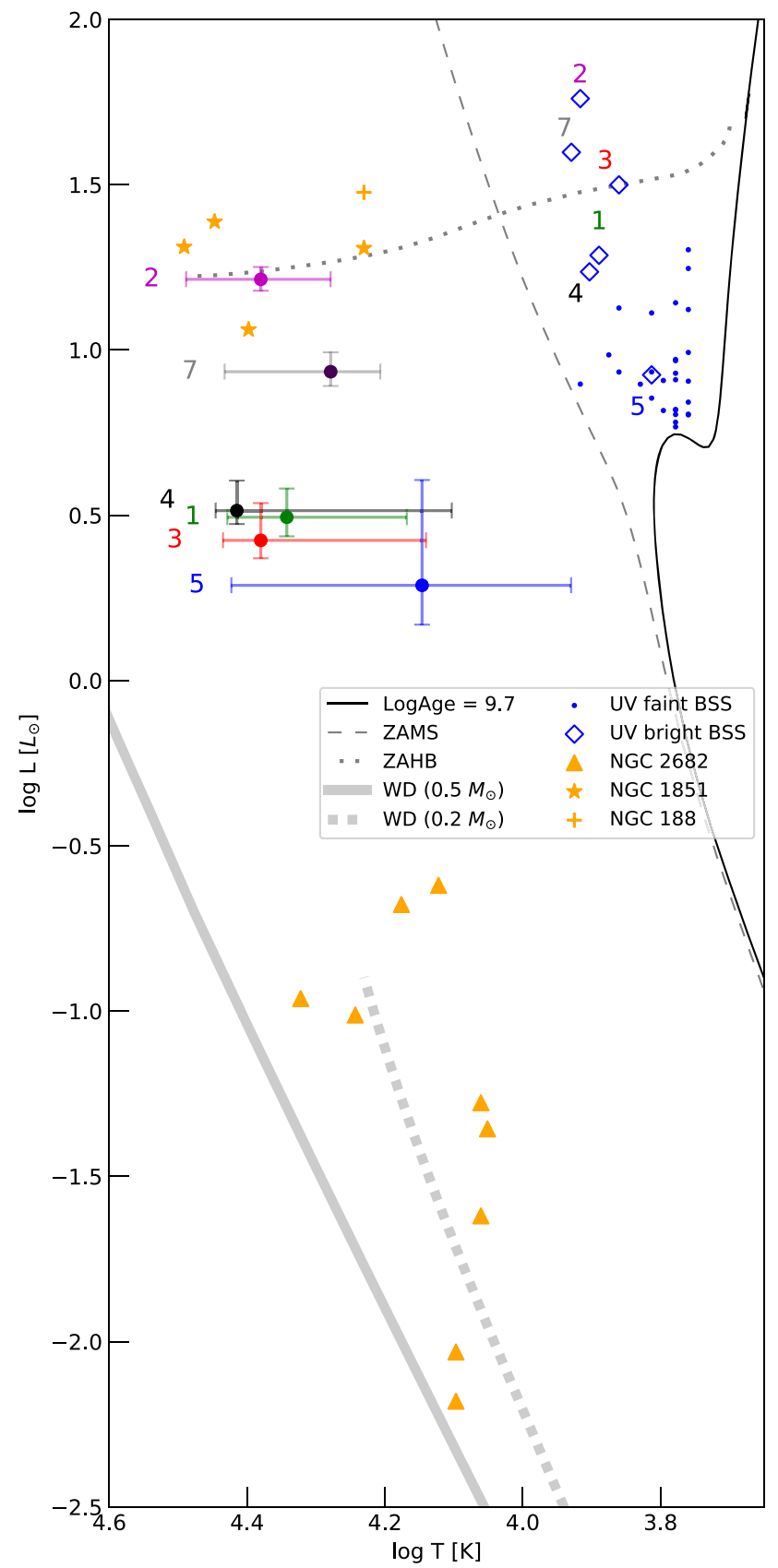

Figure 4. H-R diagram of locations of components of binaries in King 2, NGC 2682 (Sindhu et al. 2019; Jadhav et al. (2019); Sindhu et al. in prep.), NGC 188 (Subramaniam et al. 2016) and EHB stars in NGC 1851 (Singh et al. 2020). In King 2, the UV faint BSS (blue dots), UV bright BSS (blue diamonds with their ID) and hotter components in BSSs (coloured filled circles with numbers and error bars) are shown. Hotter components in NGC 2682 and NGC 188 are shown as orange triangles and cross. EHB stars in NGC 1851 are shown as stars. The PARSEC isochrone (black curve), PARSEC zero age MS (dashed grey line), WD cooling curves (thick grey curves) and BaSTI ZAHB (dotted grey curve) are shown for reference. thought to be the stripped core of a red giant star (Heber 2016). Maxted et al. (2001) found a good fraction of the sdB stars in detached, but short period binary systems. sdB stars are thought to provide important clues to common envelope evolution in tight binaries.

BSS2-B lies on the blue end of ZAHB and very similar to the EHBs in the outskirts of GC NGC 1851. Similarly, hot and bright post-AGB/HB candidate was found as a companion to a BSS in NGC 188 (Subramaniam et al. 2016). Hence, BSS2-B could be an EHB star. BSS7-B is near a known EHB from NGC 1851 and is slightly fainter than ZAHB, hence, it is likely to be an EHB. BSS5-B lies slightly above the WDs. Hence, it can be a very young He-WD (Panei et al. 2007) or an sdB star. Rest of the King 2 BSS companions are slightly fainter than the ZAHB, and they are likely sdBs.

\section{Formation pathways of BSSs and EHBs/sdBs}

BSS formation mechanism involves mass gain while the $\mathrm{EHB} / \mathrm{sdB}$ formation involves the stripping of the envelope of a post-MS star. The detection of EHB/ sdBs confirms 'binary mass transfer' as the formation mechanism for BSS1 to BSS5 and BSS7. As the cooler companions are BSSs that are supposed to have gained mass, we can infer that the detected EHBs/sdBs have transferred mass to the BSSs companions. Therefore, the $\mathrm{BSS}+\mathrm{EHB} / \mathrm{sdB}$ systems in King 2 illustrate stars on both sides of the mass exchange. We see a range in their temperature and luminosity, suggesting a diversity among the hotter companions.

The lifespan of sdB stars is expected to be between 100 to $200 \mathrm{Myr}$ (Bloemen et al. 2014; Schindler et al. 2015 and references therein), after which they descend the WD cooling curve. When the current BSS expands, the mass transfer is expected to start again due to the short orbit, which already allowed the previous instance of mass transfer. The system can begin stable/unstable mass transfer and become a WD+WD system. Alternatively, it can merge through a common-envelop phase and become a massive WD. The exact evolution will depend on then orbital parameters, mass transfer efficiency and mass loss.

King 2 is one of the oldest OC, lies in the outskirts of the galactic disk. It is metal-poor compared to the Galactic disc OCs. The environment is quite similar to outskirts of GCs, which are also metal-poor, old and 
of comparable density. While most of the BSSs in GCs lie in core and are formed via mergers (Chatterjee et al. 2013), BSSs in outskirts of GCs can form through mass transfer as seen in EHB-4 of NGC 1851 (Singh et al. 2020). Our study suggests that the at least $15 \%$ of the BSSs in King 2 are formed via mass transfer pathway of formation.

We have seen sdB companions to BSSs in NGC 188 and NGC 1851 (both are older systems), however none in NGC 2682 (which is younger). NGC 6791 of slightly younger age also has sdB stars (Kaluzny \& Udalski 1992; Reed et al. 2012). This might suggest that there is an upper age limit of $\sim 5 \mathrm{Gyr}$ for the formation of $\mathrm{sdB}$ stars in OCs.

\section{Conclusions and summary}

- The old OC King 2 has a large population (39) of BSSs, spreading up to 3 mag brighter than the MSTO. We constructed SEDs of all the BSS using UV to IR data. The BSSs have $T_{\text {eff }}$ in the range of 5750-8250 K, luminosity in the range of $5.6-57.5 L_{\odot}$ and mass in the range of $1.2-$ $1.9 M_{\odot}$.

- Six of the UV bright BSS showed excess UV flux and were successfully fitted with double component SEDs. The hotter components have $T_{\text {eff }}$ of $14000-26000 \mathrm{~K}$ and $R / R_{\odot}$ of $0.09-0.27$, suggesting a range of properties. Two of the hotter companions to the BSS are likely EHB stars, while four are likely sdB stars.

- EHB/sdB companions imply that these 6 (out of 39) BSSs have formed via binary mass transfer. The SED fits show that sdB stars can be created in old OCs such as King 2 (similar to old OC NGC 188 and GC NGC 1851).

Spectroscopic time series and radial velocity variations can uncover the binary nature as well as properties of these systems and help in estimating the log $g$ and mass of these stars. The mass and orbital estimations will expand our knowledge of BSS, EHB and $\mathrm{sdB}$ formation scenarios.

\section{Acknowledgements}

We thank the anonymous referee for their valuable comments and inputs. RS would like to thank the National Academy of Sciences, India (NASI), Prayagraj for the award the NASI Honorary Scientist; Alexander von
Humboldt Foundation, Germany for the award of longterm group research linkage program and Director, IIA for hosting and making available facilities of the institute. This publication uses the data from the AstroSat mission of the Indian Space Research Organisation (ISRO), archived at the Indian Space Science Data Centre (ISSDC). This publication uses UVIT data processed by the payload operations centre at Indian Institute of Astrophysics (IIA). The UVIT is built in collaboration between IIA, Inter-University Centre for Astronomy and Astrophysics (IUCAA), Tata Institute of Fundamental Research (TIFR), ISRO and Canadian Space Agency (CSA). This work has made use of data from the European Space Agency (ESA) mission Gaia (https:// www.cosmos.esa.int/gaia), processed by the Gaia Data Processing and Analysis Consortium (DPAC, https:// www.cosmos.esa.int/web/gaia/dpac/consortium). This publication makes use of VOSA, developed under the Spanish Virtual Observatory project supported by the Spanish MINECO through grant AyA2017-84089.

\section{References}

Ahumada J. A., Lapasset E. 2007, A\&A, 463, 789

Andrae R., Schulze-Hartung T., Melchior P. 2010, arXiv e-prints, arXiv:1012.3754

Aparicio A., Bertelli G., Chiosi C., Garcia-Pelayo J. M. 1990, A\&A, 240, 262

Bayo A., Rodrigo C., Barrado Y., Navascués D. et al. 2008, A\&A, 492, 277

Bianchi L., GALEX Team 2000, Memorie della Società Astronomia Italiana, 71, 1123

Bloemen S., Hu H., Aerts C. et al. 2014, A\&A, 569, A123

Bond H. E., Perry C. L. 1971, Pub. Astron. Soc. Pac., 83, 638

Bressan A., Marigo P., Girardi L., Salasnich B., Dal Cero C., Rubele S., Nanni A. 2012, MNRAS, 427, 127

Brown T. M., Ferguson H. C., Davidsen A. F., Dorman B. 1997, ApJ, 482, 685

Cantat-Gaudin T., Jordi C., Vallenari et al. 2018, A\&A, 618, A93

Castelli F., Gratton R. G., Kurucz R. L. 1997, A\&A, 318, 841

Chambers K. C., Magnier E. A., Metcalfe N. et al. 2016, arXiv e-prints, arXiv:1612.05560

Chatterjee S., Rasio F. A., Sills A., Glebbeek E. 2013, ApJ, 777, 106

Dias W. S., Alessi B. S., Moitinho A., Lépine J. R. D. 2002, A\&A, 389, 871

Fitzpatrick E. L. 1999, Pub. Astron. Soc. Pac., 111, 63

Friel E. D., Janes K. A., Tavarez M., Scott J., Katsani R., Lotz J., Hong L., Miller N. 2002, AJ, 124, 2693

Gaia Collaboration, Brown A. G. A., Vallenari A. et al. 2018, A\&A, 616, A1 
Gaia Collaboration, Brown A. G. A., Vallenari A. et al. 2020, arXiv e-prints, arXiv:2012.01533

Geller A. M., Mathieu R. D. 2011, Nature, 478, 356

Gosnell N. M., Mathieu R. D., Geller A. M., Sills A., Leigh N., Knigge C. 2014, ApJL, 783, L8

Gosnell N. M., Mathieu R. D., Geller A. M., Sills A., Leigh N., Knigge C. 2015, ApJ, 814, 163

Han Z., Podsiadlowski P., Maxted P. F. L., Marsh T. R., Ivanova N. 2002, MNRAS, 336, 449

Heber U. 2016, Pub. Astron. Soc. Pac., 128, 082001

Heggie D. C. 1975, MNRAS, 173, 729

Hidalgo S. L., Pietrinferni A., Cassisi S. et al. 2018, ApJ, 856,125

Indebetouw R., Mathis J. S., Babler B. L. et al. 2005, ApJ, 619, 931

Jadhav V. V., Sindhu N., Subramaniam A. 2019, ApJ, 886, 13

Jadhav V. V., Pennock C. M., Subramaniam A., Sagar R., Nayak P. K. 2021, MNRAS, 503, 236

Kaluzny J. 1989, Acta Astron., 39, 13

Kaluzny J., Udalski A. 1992, Acta Astron., 42, 29

Kozai Y. 1962, AJ, 67, 591

Kumar A., Ghosh S. K., Hutchings J. et al. 2012, SPIE, 8443, 84431N

Leiner E., Mathieu R. D., Vanderburg A., Gosnell N. M., Smith J. C. 2019, ApJ, 881, 47

Marsh T. R., Dhillon V. S., Duck S. R. 1995, MNRAS, 275, 828

Maxted P. F. L., Heber U., Marsh T. R., North R. C. 2001, MNRAS, 326, 1391

Panei J. A., Althaus L. G., Chen X., Han Z. 2007, MNRAS, 382, 779

Postma J. E., Leahy D. 2017, Pub. Astron. Soc. Pac., 129, 115002
Rani S., Subramaniam A., Pandey S., Sahu S., Mondal C., Pandey G. 2021, J. Astrophys. Astr., 42, https://doi.org/ 10.1007/s12036-020-09683-2

Reed M. D., Baran A., Tensen R. H., Telting J., O’Toole S. J. 2012, MNRAS, 427, 1245

Rucinski S. M. 1998, AJ, 116, 2998

Sahoo S. K., Baran A. S., Heber U. et al. 2020, MNRAS, 495, 2844

Sahu S., Subramaniam A., Simunovic M. et al. 2019, ApJ, 876, 34

Schindler J.-T., Green E. M., Arnett W. D. 2015, ApJ, 806, 178

Sindhu N., Subramaniam A., Jadhav V. V. et al. 2019, ApJ, 882, 43

Singh G., Sahu S., Subramaniam A., Yadav R. K. S. 2020, ApJ, 905, 44

Skrutskie M. F., Cutri R. M., Stiening R. et al. 2006, AJ, 131,1163

Subramaniam A., Sindhu N., Tandon S. N. et al. 2016, ApJL, 833, L27

Subramaniam A., Pandey S., Jadhav V. V., Sahu S. 2020, J. Astrophys. Astr., 41, 45

Tadross A. L. 2001, New Astronomy, 6, 293

Tandon S. N., Hutchings J. B., Ghosh S. K. et al. 2017, J. Astrophys. Astr., 38, 28

Taylor M. B. 2005, Astronomical Data Analysis Software and Systems XIV, 347, 29

Tody D. 1993, Astronomical Data Analysis Software and Systems II, 52, 173

Tremblay P.-E., Bergeron P. 2009, ApJ, 696, 1755

Warren S. R., Cole A. A. 2009, MNRAS, 393, 272

Wright E. L., Eisenhardt P. R. M., Mainzer et al. 2010, AJ, 140,1868 